\title{
First-principles calculation of the Andreev conductance of carbon wires
}

\author{
Bin Wang, ${ }^{1}$ Yadong Wei, ${ }^{2}$ and Jian Wang ${ }^{1, *}$ \\ ${ }^{1}$ Department of Physics and the Center of Theoretical and Computational Physics, The University of Hong Kong, \\ Pokfulam Road, Hong Kong, China \\ ${ }^{2}$ Department of Physics, School of Science Shenzhen University, Shenzhen 518060, China
}

(Received 14 February 2012; revised manuscript received 18 May 2012; published 11 July 2012)

\begin{abstract}
We developed a first-principles approach based on nonequilibrium Green's function (NEGF) combined with density functional theory (DFT) to investigate quantum transport properties of normal-metal-superconductor (N-S) hybrid systems. As an application of our theory, we investigated the Andreev conductance of atomic wires consisting of 4-15 carbon atoms in contact with one normal Al lead and another superconducting Al lead from first principles. Numerical results show that the Andreev conductance oscillates between an even and odd number of carbon atoms. In the presence of the superconducting lead, the self-consistent scattering potential of the N-S system can be very different from that of the corresponding normal system. Furthermore, a small change of scattering potential can give rise to a significant change of Andreev conductance. For an even number of carbon atoms, the change of scattering potential gives rise to a 4-7\% difference in conductance, while when the number of carbon atoms $n$ is odd, a $14-30 \%$ change of conductance is observed due to the potential change. We find that the charge transfer plays an important role in N-S systems. For the carbon wire with normal Al contacts, there is a significant charge transfer in real space that is responsible for the even-odd oscillation in conductance. When a superconducting lead is present, the charge is redistributed in momentum space, although it is almost not changed in real space. For even $n$, a $10 \%$ change of charge density at Fermi level is found mainly in the lead region. For odd $n$, however, the change of charge density at Fermi level is even more than $30 \%$ near the first, third, etc., carbon atoms. Since less charge density is available at Fermi level, there is a decrease in conductance for all carbon wires, especially for the wires with odd number of carbon atoms. Our results indicate that the self-consistent calculation of the scattering potential is necessary to obtain an accurate Andreev conductance of $\mathrm{N}-\mathrm{S}$ hybrid structures.
\end{abstract}

DOI: 10.1103/PhysRevB.86.035414

PACS number(s): 85.35.-p, 73.63.-b, 71.15.Mb

\section{INTRODUCTION}

Normal-metal-superconductor (N-S) hybrid structures have attracted continuous attention in the past few decades motivated by their broad application prospect in superconducting nanoelectronics, spintronics, as well as quantum logic circuits based on superconductors. ${ }^{1-6}$ The quantum physics associated with the coherence transport properties in N-S hybrid structures has been extensively investigated theoretically and experimentally. ${ }^{7,8}$ At the N-S interface, Andreev reflection occurs, where an electron in the normal metal is reflected as a hole and a Cooper pair appears in the superconductor. ${ }^{9}$ Due to the Andreev reflection, the conductance quantum of the N-S hybrid structure is twice that of the normal conductance quantum. Recently, a superconducting singlemolecular transistor was realized in low- dimensional carbon structures, such as graphene, nanotube, and fullerene. ${ }^{10-12}$ The transport properties were studied for the first time in N-S single-fullerene-molecular transistor. ${ }^{12}$ By adjusting the magnitude of gate voltage and magnetic field, it was demonstrated that the effects of Coulomb repulsion, Kondo correlations, and superconductivity can coexist and compete in the $C_{60}$ molecule over a broad range of coupling strengths of the device. Although many theoretical formalisms have been developed to describe the transport properties in various low-dimensional hybrid mesoscopic systems, such as N-S-N, S-S-N, N-N-S, and S-N-S, ${ }^{13-19}$ a first-principles quantum transport theory needed to predict transport properties of molecular devices is yet to be developed. It is the purpose of this paper to fill this gap.
In the presence of N-S interface, Beenakker presented a general theory to describe the phase-coherent multichannel Andreev conductance based on the random-matrix theory. ${ }^{13}$ The Andreev conductance $G_{N S}$ of the N-S system is related to the transmission coefficient of the normal system. The electron transport through N-S system was separated into two processes: the transport through the central scattering region (normal region) that is characterized by a scattering matrix $s_{N}$ and the Andreev reflection at N-S interface characterized by Andreev reflection coefficient $s_{0}$ that changes an electron to a hole. The combination of these two matrices determines the Andreev conductance of the hybrid system that is given by (at zero temperature $)^{13}$

$$
G_{N S}=\frac{4 e^{2}}{h} \sum_{m=1}^{N} \frac{T_{m}^{2}}{\left(2-T_{m}\right)^{2}},
$$

where $T_{m}$ is the transmission coefficient of the $m$ th eigenchannel in the normal state. In the theory of Beenakker an implicit assumption was made, i.e., the scattering potential is not affected by the presence of the superconducting lead. This is a good approximation in mesoscopic systems. In fact, this theory has been used to analyze the experimental data in N-S point contacts and obtained qualitatively agreement. ${ }^{4,20}$ Therefore, if the superconducting lead does not modify the scattering potential, one can use Eq. (1) to calculate the Andreev conductance of the N-S system. However, it has been emphasized that internal Coulomb potential in the N-S hybrid system must be included in order to keep the gauge invariance. ${ }^{21}$ 
As we will show in this paper, this Coulomb potential is very sensitive to the presence of the superconducting lead due to the charge transfer. There is a significant change in Andreev conductance due to the change of effective potential when a superconducting lead is present. Hence we should calculate the Coulomb potential self-consistently especially for the N-S molecular devices.

In this paper, we propose a theory that combines the nonequilibrium Green's function with the density functional theory to predict transport properties of molecular N-S junctions. This theory allows us to determine the Coulomb potential self-consistently so that the gauge invariance is ensured. As an application, we have calculated the Andreev conductance of Al- $\mathrm{C}_{n}-\mathrm{Al}$ hybrid $\mathrm{N}-\mathrm{S}$ structures from first principles and compared the results with those from Eq. (1). Our numerical result shows that a change of Coulomb potential due to the superconducting lead on an odd number of carbon wires is more significant than that of an even number of carbon wires. Although the charge density does not change very much in real space when the superconducting lead is present, there is a significant redistribution of charge density in energy or momentum space which gives rise to a large decrease of density of states at the Fermi level. For instance, the charge density at the Fermi level can have over $30 \%$ change for an odd number of carbon wires. Finally, we find that the Andreev conductance oscillates between an even and odd number of carbon atoms.

The remainder of the paper is organized as follows. In Sec. II, our transport theory of N-S hybrid structures is presented. In Sec. III, this theory is used to calculate the Andreev conductance in atomic structures. We also give a detailed analysis for numerical results. Section IV is a summary of this work.

\section{THEORETICAL FORMALISM}

In this section, we present the gauge-invariant nonlinear transport theory based on nonequilibrium Green's function (NEGF) for the N-S hybrid system. Since the theory is gauge invariant, we fix $v_{R}=0$ and the bias difference is $v=v_{L}$. The Hamiltonian of a N-S hybrid system can be written as ${ }^{16,22,23}$ $(e=\hbar=1)$

$$
\begin{aligned}
H= & \sum_{k, \sigma}\left(\epsilon_{L, k}-v_{L}\right) a_{L, k \sigma}^{\dagger} a_{L, k \sigma}+\sum_{k, \sigma} \epsilon_{R, k} a_{R, k \sigma}^{\dagger} a_{R, k \sigma} \\
& +\sum_{k}\left[\Delta_{k}^{*} a_{R, k \downarrow} a_{R,-k \uparrow}+\Delta_{k} a_{R,-k \uparrow}^{\dagger} a_{R, k \downarrow}^{\dagger}\right] \\
& +\sum_{n, \sigma}\left(\epsilon_{n}-V_{n}\right) d_{n \sigma}^{\dagger} d_{n \sigma} \\
& +\sum_{k \alpha, n \sigma}\left[t_{k \alpha n \sigma} a_{\alpha, k \sigma}^{\dagger} d_{n \sigma}+t_{k \alpha n \sigma}^{*} d_{n \sigma}^{\dagger} a_{\alpha, k \sigma}\right],
\end{aligned}
$$

where the first term in this equation describes the Hamiltonian of the left normal lead; the second and third terms describe the Hamiltonian of the right superconducting lead with $\Delta_{k}$ the superconductor pair potential in the $k$ state; the fourth term is the Hamiltonian of the central scattering region; and the last term gives the coupling between the leads and the scattering region. It should be noted that the pair potential $\Delta_{k}=$ $-V_{0}\left\langle a_{R, k \downarrow} a_{R,-k \uparrow}\right\rangle$ is real-space dependent with $V_{0}$ a positive electron-phonon interaction which leads to superconductivity, $\Delta(\mathbf{r})=\Delta$ inside the superconducting lead and $\Delta(\mathbf{r})=0$ inside the normal lead. Near the N-S interface within the phase coherent length, $\Delta(\mathbf{r})$ should be determined by self-consistent calculation to keep gauge invariance. ${ }^{17,24} \mathrm{~A}$ self-consistent calculation of pair potential has been investigated in Ref. 25 for superconducting mesoscopic weak links. However, due to the technical difficulty of $a b$ initio calculation, $\Delta(\mathbf{r})$ was replaced with a step function in our calculation and only the internal potential $V_{n}$ was calculated self-consistently. The internal potential $V_{n}$ consists of Hartree potential $V_{h}$ and exchange-correlation potential $V_{x c}$ where $V_{h}$ is determined by solving the Poisson equation, ${ }^{21}$

$$
\nabla^{2} V_{h}(\mathbf{r})=-4 \pi i \int \frac{d E}{2 \pi}\left[G_{11}^{<}(E, U(\mathbf{r}))\right],
$$

where $G_{11}^{<}$is the electron lesser Green's function. According to Ref. $22, G_{11}^{<}$can be calculated by

$$
\begin{aligned}
G_{11}^{<}= & i \int \frac{d E}{2 \pi}\left[G_{11}^{r} \Gamma_{L} G_{11}^{a} f_{L}\left(E+v_{L}\right)\right. \\
& \left.+G_{12}^{r} \Gamma_{L} G_{21}^{a} f_{L}\left(E-v_{L}\right)\right] \\
& +i \int \frac{d E}{2 \pi} \rho_{R}(E) f_{R}(E)\left[G_{11}^{r} \Gamma_{R} G_{11}^{a}+G_{12}^{r} \Gamma_{R} G_{21}^{a}\right. \\
& \left.-\frac{\Delta}{|E|}\left(G_{11}^{r} \Gamma_{R} G_{21}^{a}+G_{12}^{r} \Gamma_{R} G_{11}^{a}\right)\right]
\end{aligned}
$$

Here $G_{11}^{r}$ and $G_{12}^{r}$ are the matrix elements in $2 \times 2$ Nambu representation that are given by Eqs. (4) and (6) in Ref. 21 The self-energy $\Sigma^{r}=\Sigma_{L}^{r}+\Sigma_{R}^{r}$ which is a $2 \times 2$ matrix in spin space is given by ${ }^{22}$

$$
\Sigma_{L}^{r}(E)=\left(\begin{array}{cc}
\Sigma_{L}^{r}(E) & 0 \\
0 & -\Sigma_{L}^{a}(-E)
\end{array}\right),
$$

where $\Sigma_{\alpha}^{r} \equiv P_{\alpha}-i \Gamma_{\alpha} / 2$ is the self-energy of lead $\alpha$ in the normal case. Here $P_{\alpha}$ and $-\Gamma_{\alpha} / 2$ are the real and imaginary parts of the self-energy $\Sigma_{\alpha}^{r}$ of the atomic lead $\alpha$ in the normal state which must be calculated from the first principles. We see that the self-energy of the left normal lead is diagonal in spin space, while for the right superconducting lead, the off diagonal matrix elements of self-energy are nonzero. In the Andreev approximation where $|\Delta|, E \ll E_{F}$, we have ${ }^{26}$

$$
\Sigma_{R}^{r}=\left(\begin{array}{cc}
P_{R}-i \frac{\Gamma_{R}}{2} \beta_{1} & i \frac{\Gamma_{R}}{2} \beta_{2} \\
i \frac{\Gamma_{R}}{2} \beta_{2} & -P_{R}-i \frac{\Gamma_{R}}{2} \beta_{1}
\end{array}\right),
$$

where $\beta_{1}=v E / \sqrt{E^{2}-\Delta^{2}}$ and $\beta_{2}=v \Delta / \sqrt{E^{2}-\Delta^{2}}, v=1$ when $E>-\Delta$, and $v=-1$ otherwise. The dimensionless $\mathrm{BCS}$ density of states (DOS) is given by $\rho_{R}=\theta(|E|-\Delta) \beta_{1}$. Equations (3) and (4) form the basic equations to describe the nonlinear transport properties of N-S systems. We notice that the self-consistent calculation has to be done in Nambu presentation that involves the coupling between electron and hole. This is quite different from the case when the right lead is in the normal state.

After the self-consistent solution from Eqs. (3) and (4) is obtained, the Green's function and scattering potential landscape denoted as $V_{N S}$ can be used to calculate the Andreev current $I_{L}$ through the normal lead [see Eqs. (1)-(3) of Ref. 21]. 
If the bias voltage is less than $\Delta$, the Andreev conductance is given by

$$
G_{N S}=\frac{4 e^{2}}{h} \int d E\left(-\partial_{E} f_{L}\right) \operatorname{Tr}\left[\Gamma_{L}(E) G_{12}^{r}(E) \Gamma_{L}(-E) G_{21}^{a}(E)\right] .
$$

It should be noted that if the difference of scattering potential due to the presence of superconducting lead is ignored, i.e., when $V_{N S} \approx V_{N}$ where $V_{N}$ is the scattering potential of the system with normal leads, Eq. (7) is the same as Eq. (1). As will be seen in the next section, this difference cannot be neglected in general.

\section{NUMERICAL RESULTS}

In this section, we apply our formalism to atomic N-S hybrid structures to calculate the Andreev conductance. Our numerical scheme is based on the NEGF theory for N-S hybrid systems combined with density functional theory (DFT) which is a generalization of our MCDCAL package ${ }^{27}$ to N-S hybrid systems. A linear combination of the atomic orbitals (LCAO) basis set ${ }^{28}$ was employed to solve Eqs. (3) and (4) numerically. The exchange correlation was treated at the LSDA level ${ }^{29}$ and a nonlocal norm conserving pseudopotential ${ }^{30}$ was used to define the atomic core. The density matrix was constructed in orbital space and the internal potential was solved in real space. In our calculation, the NEGF-DFT self-consistency was carried out until the numerical tolerance was less than $10^{-4} \mathrm{eV}$.

Specifically, we study the structures of one-dimensional carbon wires in contact with a normal $\mathrm{Al}$ lead and a superconducting $\mathrm{Al}$ lead $\left(\mathrm{Al}-\mathrm{C}_{n}-\mathrm{Al}\right)$ with $n$ from 4 to 15 . The schematic structure of $\mathrm{Al}-\mathrm{C}_{6}-\mathrm{Al}$ is shown in Fig. 1. The two semi-infinite leads consist of a periodically repeated unit cell (including nine atoms) along the (100) direction to $x= \pm \infty$. In our calculation, the distance between the adjacent two carbon atoms is 2.5 a.u. and between the $\mathrm{Al}$ slab and carbon atom is fixed to 3.78 a.u.

As we have discussed in the Introduction, two scattering potentials for $\mathrm{Al}-\mathrm{C}_{n}-\mathrm{Al}$ system can be calculated using the NEGF-DFT method: $V_{N}$ is the potential when the right lead is in the normal state, and $V_{N S}$ is the potential when the right lead is in the superconducting state. For $V_{N}$, the normal transmission coefficient $T_{n}$ can be calculated. This in turn gives the Andreev conductance (denoted as $\bar{G}_{N S}$ ) from Eq. (1). For $V_{N S}$, we calculate the Andreev conductance (denoted as $G_{N S}$ ) from Eq. (7). Clearly, $G_{N S}$ is the accurate result while $\bar{G}_{N S}$ is an approximate result by assuming $V_{N S} \approx V_{N}$.

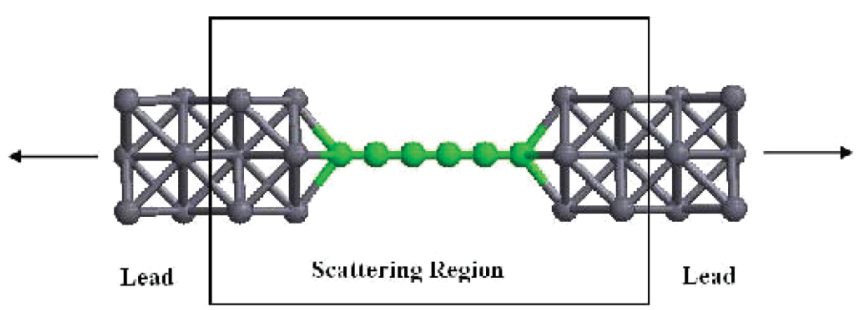

FIG. 1. (Color online) $\mathrm{Al}-\mathrm{C}_{6}-\mathrm{Al}$ structure where an atomic wire with six carbon atoms is sandwiched between a normal $\mathrm{Al}$ lead and a superconducting $\mathrm{Al}$ lead. The frame is the simulation box.
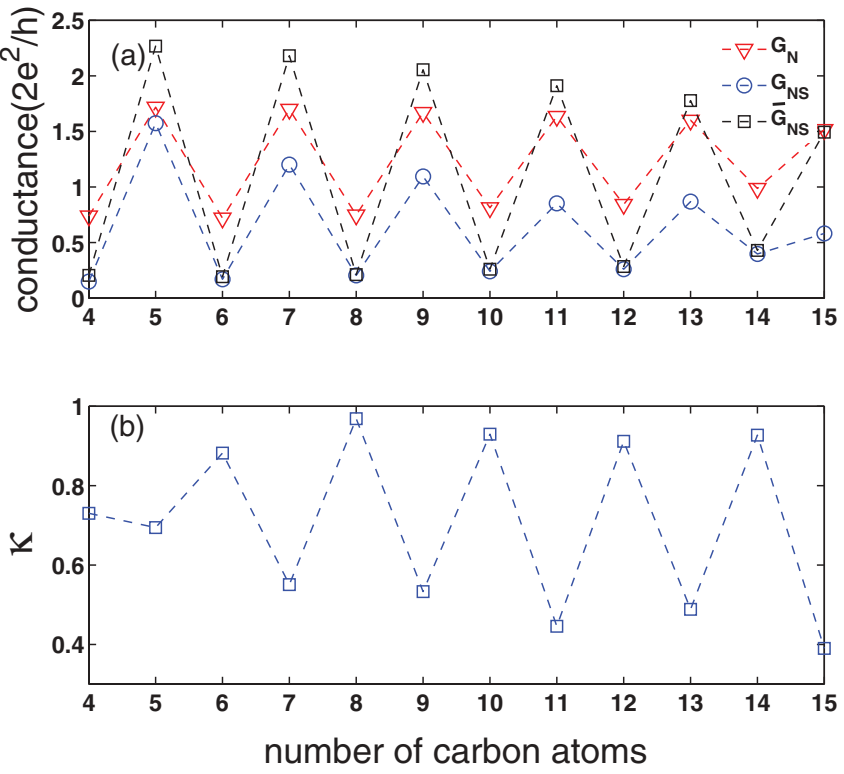

FIG. 2. (Color online) (a) Conductance oscillation with the number of carbon atoms $n$ of $\mathrm{Al}-\mathrm{C}_{n}-\mathrm{Al}$ systems, where $n=4,5,6, \ldots, 15$. The red triangles, black squares, and blue circles correspond to $G_{N}$, $\bar{G}_{N S}$, and $G_{N S}$, respectively. (b) $\kappa=G_{N S} / \bar{G}_{N S}$ versus $n$ for $\mathrm{Al}-\mathrm{C}_{n}-\mathrm{Al}$ systems.

In this paper, the Andreev conductance of $\mathrm{Al}-\mathrm{C}_{n}-\mathrm{Al}$ hybrid structures with $n=4$ to 15 were calculated using Eqs. (1) and (7). The conductance of normal system can be obtained by $G_{N}=2 e^{2} / h \sum_{n} T_{n}$.

In Fig. 2(a) we plot $G_{N}, \bar{G}_{N S}$, and $G_{N S}$ versus the number of carbon atoms $n$ so that the effect of the superconducting lead to the scattering potential can be examined. The following observations are in order: (1) For the normal system, the conductance $G_{N}$ shows oscillatory behavior for an even and odd number of carbon atoms. This feature has been observed in mesoscopic systems for normal and NS systems before. ${ }^{31}$ From the first-principles calculation ${ }^{32}$ it is attributed to charge transfer from $\mathrm{Al}$ lead to the carbon atoms. (2) When the right lead becomes superconducting, $\bar{G}_{N S}$ and $G_{N S}$ also oscillate with an even and odd number of carbon atoms. (3) For the carbon wires with an even number of atoms the Andreev conductance $\bar{G}_{N S}$ and $G_{N S}$ are very close for each $n$ and dropped by about $0.5 \times 2 e^{2} / h$ compared with the conductance $G_{N}$. This means that $V_{N S}=V_{N}$ is not a bad approximation for even $n$. (4) For an odd number of carbon atoms, the situation is different. The Andreev conductance $\bar{G}_{N S}$ has a big increase over $G_{N}$ for the wire with five carbon atoms $C_{5}$. The difference $\bar{G}_{N S}-G_{N}$ decreases for longer wires. The longer the wire, the more is the decrease. When $n=15$, we have $\bar{G}_{N S} \approx G_{N}$. On the other hand, the Andreev conductance $G_{N S}$ is smaller than but close to $G_{N}$ for $n=5$. The difference $G_{N S}-G_{N}$ increases for larger $n$. To see the difference between $G_{N S}$ and $\bar{G}_{N S}$ clearly, the ratio $\kappa=G_{N S} / \bar{G}_{N S}$ versus the number of atoms $n$ is shown in Fig. 2(b). We see that $\kappa$ is close to 0.9 for carbon wires with an even number of atoms and decreases for an odd number of atoms from 0.7 to 0.35 . From Fig. 2 we conclude that Eq. (1) overestimates Andreev conductance for the odd $n$ by a large amount. Obviously, this is due to the change of scattering potential when the 


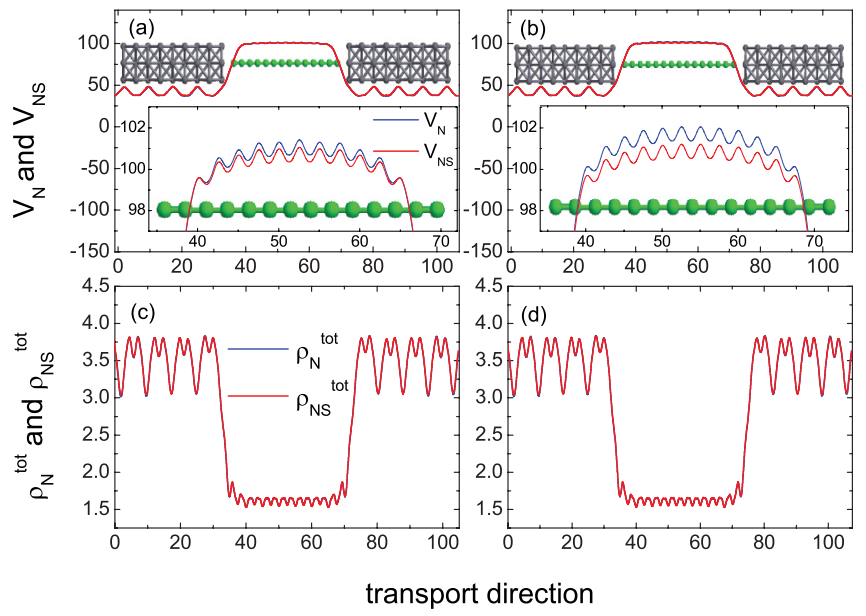

FIG. 3. (Color online) (a),(b) Scattering potentials $V_{N}$ and $V_{N S}$ and (c),(d) charge distribution $\rho_{N}^{\text {tot }}$ and $\rho_{N S}^{\text {tot }}$ along the transport direction for $\mathrm{Al}-\mathrm{C}_{14}-\mathrm{Al}$ and $\mathrm{Al}-\mathrm{C}_{15}-\mathrm{Al}$, respectively. In each panel, the scattering potential or charge distribution is summed up perpendicular to the transport direction. The filled circles in each panel indicate the coordinates of atoms in the device along the transport direction.

superconducting lead is present. To see this point more clearly, we calculated the normal conductance $\bar{G}_{N}$ for all Al-C ${ }_{n}-\mathrm{Al}$ structures. $\bar{G}_{N}$ is calculated by $\bar{G}_{N}=2 e^{2} / h \sum_{n} \bar{T}_{n}$ where $\bar{T}_{n}$ is the transmission coefficient due to the superconducting scattering potential $V_{N S}$. We found that $\bar{G}_{N}$ is always smaller than the corresponding $G_{N}$ for all the atomic wires. In addition, the conductance difference is larger for the structures with an odd number of carbon atoms. As a result, it is necessary to recalculate the scattering potential self-consistently when a normal lead is replaced by a superconducting lead.

To understand the large difference between $\bar{G}_{N S}$ and $G_{N S}$, we try to compare the scattering potential $V_{N}$ for normal systems and $V_{N S}$ for N-S systems of all the atomic wires in the real space. We found that there is a slight difference between $V_{N}$ and $V_{N S}$ for all the carbon wires with the number of atoms from 4 to 15 . To show that $V_{N}$ and $V_{N S}$ of $\mathrm{Al}-\mathrm{C}_{14}-\mathrm{Al}$ and $\mathrm{Al}-\mathrm{C}_{15}-\mathrm{Al}$ structures are plotted along the device direction in Figs. 3(a) and 3(b), respectively, where the scattering potential is summed up on each slice perpendicular to the transport direction. We see that the potential difference $d V$ for even $n$ is very small, while for odd $n, d V$ is relatively larger. It is a little surprising that such a small $d V$ can induce a large difference between $\bar{G}_{N S}$ and $G_{N S}$.

Physically, the change of scattering potential due to the presence of superconducting lead will affect the distribution of charge density. We have calculated the real-space distribution of total charge $\rho_{N}^{\text {tot }}(\mathbf{r})$ from $V_{N}$ and $\rho_{N S}^{\text {tot }}(\mathbf{r})$ from $V_{N S}$ of all the atomic structures. $\rho_{N}^{\text {tot }}(\mathbf{r})$ and $\rho_{N S}^{\text {tot }}(\mathbf{r})$ can be calculated as follows:

$$
\rho_{\alpha}^{\mathrm{tot}}(\mathbf{r})=\int d E f(E) \rho_{\alpha}^{E}(\mathbf{r})=\int d E f(E)[\operatorname{DOS}(E)]_{\mathbf{r}, \mathbf{r}},
$$

where the subscript $\alpha=N$ or $N S . \rho_{\alpha}^{E}(\mathbf{r})$ is the charge distribution at a particular energy and DOS is the corresponding local density of states. The numerical result shows that the difference between $\rho_{N}^{\text {tot }}$ and $\rho_{N S}^{\text {tot }}$ is very small at each real-space grid for all structures. In Figs. 3(c) and 3(d), both $\rho_{N}^{\text {tot }}$ and
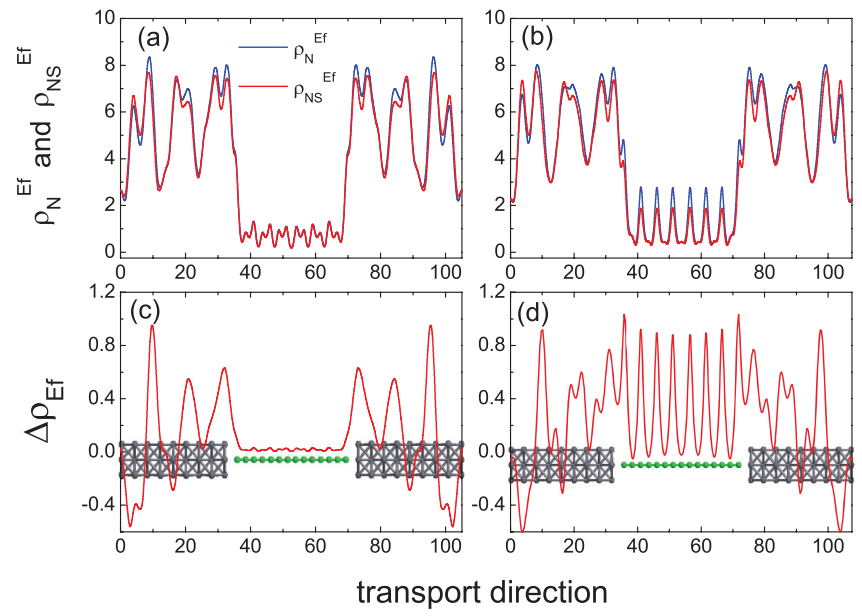

FIG. 4. (Color online) (a),(b) Real-space charge distribution at Fermi level $\rho_{N}^{E f}$ and $\rho_{N S}^{E f}$ for $\mathrm{Al}-\mathrm{C}_{14}-\mathrm{Al}$ and $\mathrm{Al}-\mathrm{C}_{15}-\mathrm{Al}$ structures, respectively. (c),(d) $\Delta \rho_{E f}=\rho_{N}^{E f}-\rho_{N S}^{E f}$ along the transport direction for $\mathrm{Al}-\mathrm{C}_{14}-\mathrm{Al}$ and $\mathrm{Al}-\mathrm{C}_{15}-\mathrm{Al}$ structures, respectively. For each panel, $\rho_{N}^{E f}$ and $\rho_{N S}^{E f}$ are summed up perpendicular to the transport direction. The filled circles in each panel indicate the coordinates of atoms in the device along the transport direction.

$\rho_{N S}^{\text {tot }}$ of $\mathrm{Al}-\mathrm{C}_{14}-\mathrm{Al}$ and $\mathrm{Al}-\mathrm{C}_{15}-\mathrm{Al}$ structures are plotted in the scattering region, where $\rho^{\text {tot }}$ is the total charge calculated on each plane perpendicular to the transport direction. Although $\rho_{N S}^{\text {tot }}$ is very close to $\rho_{N}^{\text {tot }}$ in the real space, we found that DOS at momentum space is redistributed when a normal lead is replaced by a superconducting lead. Note that for the $\mathrm{Al}-\mathrm{C}_{n}-\mathrm{Al}$ structure, conductance is mostly contributed by the lowest unoccupied molecular orbital (LUMO) state coupled with the highest occupied molecular orbital (HOMO) state and the equilibrium transport properties are mostly determined by the DOS at the Fermi level. ${ }^{33}$ Therefore, we investigated the real-space distribution of DOS at the Fermi level: $\rho_{N}^{E f}$ for structures with a normal right lead and $\rho_{N S}^{E f}$ for structures with a superconducting right lead. As an example, we plotted $\rho_{N}^{E f}$ and $\rho_{N S}^{E f}$ for $\mathrm{Al}-\mathrm{C}_{14}-\mathrm{Al}$ and $\mathrm{Al}-\mathrm{C}_{15}-\mathrm{Al}$ structures in Figs. 4(a) and 4(b), respectively. For Al- $\mathrm{C}_{14}-\mathrm{Al}$, the difference between $\rho_{N S}^{E f}$ and $\rho_{N}^{E f}$ mostly occurs in the lead region (about $10 \%$ ), while they are almost the same in the region of carbon wires. However, the behavior is very different for Al- $\mathrm{C}_{15}-\mathrm{Al}$. Except in the lead region where about a $10 \%$ change occurs between $\rho_{N}^{E f}$ and $\rho_{N S}^{E f}$, there is also a large difference in the region of carbon wires, especially for the first, third,..., fifteenth carbon atoms, and the difference is as large as 30\%. From the above discussion, we can conclude that it is this significant redistribution of charge in energy or momentum space that induces the large difference of Andreev conductance for an odd number of carbon wires, although the total charge distribution in real space is not influenced very much by the presence of the superconducting lead. This behavior has been confirmed to be a general behavior for all the carbon wires with $n$ from 4 to 15 .

Before closing, we note that we have made a number of approximations in this work that can affect the accuracy of the numerical results. On the DFT level, we have used pseudopotential approximation to replace ionic potentials 
and the influence of the core electrons in the core region. The local-density approximation has been used for exchange and correlation functional. To speed up the calculation, the Green's function has been expanded in terms of the local orbital (LCAO approach). Finally, the pair potential has been treated non-self-consistently. It would be interesting to test the accuracy of these approximations in the future.

\section{SUMMARY}

In summary, we developed a first-principles method within the framework of Keldysh NEGF to investigate the quantum transport properties of N-S hybrid systems. Using our method, we have calculated the Andreev conductance of $\mathrm{Al}-\mathrm{C}_{n}-\mathrm{Al}$ structures. In the calculation, the scattering potential can be treated at two levels. One can either use the scattering potential when both leads are in normal states or include the effect of superconducting leads on the scattering potential. We found that the Andreev conductance can be very different for these two cases. In particular, this difference in the Andreev conductance is more obvious for carbon wires with an odd number of atoms

*jianwang@hku.hk

${ }^{1}$ J. J. A. Baselmans, A. F. Morpurgo, and B. J. van Wees, Nature (London) 397, 43 (1999).

${ }^{2}$ M. Berciu, T. G. Rappoport, and B. Janko, Nature (London) 435, 71 (2005).

${ }^{3}$ K. Ohnishi, T. Kimura, and Y. Otani, Appl. Phys. Lett. 96, 192509 (2010).

${ }^{4}$ G. Grabecki, K. A. Kolwas, J. Wroel, K. Kapcia, R. Puniak, R. Jakiela, M. Aleszkiewicz, T. Dietl, G. Springholz, and G. Bauer, J. Appl. Phys. 108, 053714 (2010).

${ }^{5}$ D. J. Thurmer, C. Bufon, C. Deneke, and O. G. Schmidt, Nano Lett. 10, 3704 (2010).

${ }^{6}$ J. P. DeGrave, A. L. Schmitt, R. S. Selinsky, J. M. Higgins, D. J. Keavney, and S. Jin, Nano Lett. 11, 4431 (2011).

${ }^{7}$ C. W. J. Beenakker, Rev. Mod. Phys. 69, 731 (1997).

${ }^{8}$ B. J. van Wees, P. de Vries, P. Magnee, and T. M. Klapwijk, Phys. Rev. Lett. 69, 510 (1992); C. N. Lau, N. Markovic, M. Bockrath, A. Bezryadin, and M. Tinkham, ibid. 87, 217003 (2001); M. L. Tian, N. Kumar, S. Xu, J. Wang, J. S. Kurtz, and M. H. W. Chan, ibid. 95, 076802 (2005); Y.-J. Doh, J. A. van Dam, A. L. Roest, E. P. A. M. Bakkers, L. P. Kouwenhoven, and S. De Franceschi, Science 309, 272 (2005).

${ }^{9}$ A. F. Andreev, Sov. Phys. JETP 19, 1228 (1964).

${ }^{10}$ H. B. Heersche, P. J. Herrero, J. B. Oostinga, L. M. K. Vandersypen, and A. F. Morpurgo, Nature (London) 446, 56 (2007).

${ }^{11}$ P. Jarillo-Herrero, J. A. van Dam, and L. P. Kouwenhoven, Nature (London) 439, 953 (2006).

${ }^{12}$ C. B. Winkelmann, N. Roch, W. Wernsdorfer, V. Bouchiat, and F. Balestro, Nat. Phys. 5, 876 (2009).

${ }^{13}$ C. W. J. Beenakker, Phys. Rev. B 46, 12841 (1992).

${ }^{14}$ M. T. Tuominen, J. M. Hergenrother, T. S. Tighe, and M. Tinkham, Phys. Rev. Lett. 69, 1997 (1992); Phys. Rev. B 47, 11599 (1993).

${ }^{15}$ U. Hanke, Yu. Galperin, K. A. Chao, M. Gisselfalt, M. Jonson, and R. I. Shekhter, Phys. Rev. B 51, 9084 (1995).

${ }^{16}$ S. Ishizaka, J. Sone, and T. Ando, Phys. Rev. B 52, 8358 (1995).

${ }^{17}$ A. Levy Yeyati, J. C. Cuevas, A. Lopez-Davalos, and A. Martin-Rodero, Phys. Rev. B 55, R6137 (1997); C. J. than those with an even number of atoms. As a consequence, the scattering potential of N-S systems must be determined self-consistently to include the effect of the superconducting lead. In addition, we found that although the charge distribution in real space is very close in these two cases, the charge density in momentum space is redistributed which is responsible for the large change in Andreev conductance of carbon wires, especially for those with an odd number of atoms.

\section{ACKNOWLEDGMENTS}

This work was financially supported by Research Grant Council (Grant No. HKU 705611P) and University Grant Council (Contract No. AoE/P-04/08) of the Government of HKSAR. Y.D.W. was supported by grant from the National Natural Science Foundation of China (Grant No. 11074171). This research was conducted using the HKU Computer Centre research computing facilities that are supported in part by the Hong Kong UGC Special Equipment Grant (Grant No. SEG HKU09).
Lambert and R. Raimondi, J. Phys.: Condens. Matter 10, 901 (1998).

${ }^{18}$ R. Fazio and R. Raimondi, Phys. Rev. Lett. 80, 2913 (1998).

${ }^{19}$ Q. F. Sun, J. Wang, and T. H. Lin, Phys. Rev. B 59, 13126 (1999); Q. F. Sun, B. G. Wang, J. Wang, and T. H. Lin, ibid. 61, 4754 (2000); Y. D. Wei, J. Wang, H. Guo, H. Mehrez, and C. Roland, ibid. 63, 195412 (2001); Y. Zhu, T. H. Lin, and Q. F. Sun, ibid. 69, 121302(R) (2004).

${ }^{20}$ A. Tselev, Y. Yang, J. Zhang, P. Barbara, and S. E. Shafranjuk, Phys. Rev. B 80, 054504 (2009).

${ }^{21}$ J. Wang, Y. D. Wei, H. Guo, Q. F. Sun, and T. H. Lin, Phys. Rev. B 64, 104508 (2001)

${ }^{22}$ Q. F. Sun, J. Wang, and T. H. Lin, Phys. Rev. B 59, 3831 (1999).

${ }^{23}$ J. C. Cuevas, A. Martin-Rodero, and A. Levy Yeyati, Phys. Rev. B 54, 7366 (1996).

${ }^{24}$ P. G. De Gennes, Superconductivity of Metals and Alloys (Benjamin, New York, 1966).

${ }^{25}$ A. Levy Yeyati, A. Martin-Rodero, and F. J. Garcia-Vidal, Phys. Rev. B 51, 3743 (1995).

${ }^{26}$ M. P. Samanta and S. Datta, Phys. Rev. B 57, 10972 (1998).

${ }^{27}$ J. Taylor, H. Guo, and J. Wang, Phys. Rev. B 63, 245407 (2001); 63, 121104 (2001).

${ }^{28}$ P. Ordejón, E. Artacho, and J. M. Soler, Phys. Rev. B 53, R10441 (1996); J. M. Soler, E. Artacho, J. D. Gale, A. García, J. Junquera, P. Ordejón, and D. Sánchez-Portal, J. Phys.: Condens. Matter 14, 2745 (2002).

${ }^{29}$ U. von Barth and L. Hedin, J. Phys. C: Solid State Phys. 5, 1629 (1972); O. Gunnarsson and B. I. Lundqvist, Phys. Rev. B 13, 4274 (1976); A. K. Rajagopal, J. Phys. C: Solid State Phys. 11, L943 (1978). In this calculation, the spin degrees of freedom is frozen.

${ }^{30}$ N. Troullier and J. L. Martins, Phys. Rev. B 43, 1993 (1991).

${ }^{31}$ P. L. Pernas, A. Martin-Rodero, and F. Flores, Phys. Rev. B 41, 8553 (1990); T. S. Kim and S. Hershfield, ibid. 65, 214526 (2002).

${ }^{32}$ N. D. Lang and Ph. Avouris, Phys. Rev. Lett. 81, 3515 (1998).

${ }^{33}$ B. Larade, J. Taylor, H. Mehrez, and H. Guo, Phys. Rev. B 64, 075420 (2001) 